International Journal of Pure and Applied Mathematics

Volume 112 No. $3 \quad 2017,519-530$

ISSN: 1311-8080 (printed version); ISSN: 1314-3395 (on-line version)

url: http://www.ijpam.eu

doi: 10.12732/ijpam.v112i3.6

\title{
AN ANALYSIS OF RUMOR SPREADING MODEL WITH CONTRA PRODUCTIVE INTERVENTION
}

\author{
D. Aldila ${ }^{1}$, H.P. Paramartha ${ }^{2}$, H. Tasman ${ }^{3}$ \\ $1,2,3$ Department of Mathematics \\ Universitas Indonesia \\ Kampus UI Depok, Depok, 16424, INDONESIA
}

\begin{abstract}
A mathematical model to explain the spread of rumors in a closed human population is proposed in this article. Interventions from the government to educate people about the danger of rumor along with apprehensions of fanatical people are implemented in the model. Intervention of imprisonment which will send fanatical people to prison was assumed to have a worsening effect, i.e increasing the ability of fanatical people to persuade other people to follow their ideology. Equilibrium points and their local stability criteria are discussed. Some scenarios that might appear in reality have been shown numerically to give a better illustration of the model.
\end{abstract}

AMS Subject Classification: 37N99, 91C99

Key Words: rumor, equilibrium points, local stability

\section{Introduction}

In many countries, the spread of rumor has become a serious social problem which sometimes endangers the establishment of an organization or even countries themselves. Many extreme efforts to control the spread of rumors are already being made in those countries, such as persuasive efforts through comprehensive dialog or routine campaigns in electronic media, or even using military force.

Received: $\quad$ September 8, 2016

Revised: $\quad$ November 17, 2016

Published: $\quad$ February 8, 2017

$\S_{\text {Correspondence author }}$ (c) 2017 Academic Publications, Ltd. url: www.acadpubl.eu 
Many mathematical models have been introduced to study the dynamics of human behavior in response to the spread of rumors, such as in $[2,3,4,5,6,7$, $8,9]$. In this article, we develop a model based on a previous model introduced by the authors in [1]. In [1], the human population was divided into five subpopulations, i.e. a general subpopulation $S(t)$, a semi-fanatical subpopulation $E(t)$, a fanatical subpopulation $F(t)$, an aware subpopulation $A(t)$ and also a repented subpopulation $R(t)$. An intervention from the government with a mass campaign about the dangers of the rumor and also involving the arrest of fanatical people was included in the model. In the model, it was found that the more fanatical people arrested, the better the control of the spread of rumor. Unfortunately, in many circumstances there are worsening effects of this intervention. The fervor of fanatical people who have not been arrested yet in spreading their ideology more intensively is one example. In this article, this fact will be included in the model.

In the next section, the mathematical assumptions for model construction will be discussed, followed by analysis of the mathematical model in the third section. Some numerical simulation will be performed in the fourth section and the paper will close with conclusions in the last section.

\section{Model Construction}

In this chapter, the mathematical model construction to understand how rumors spread will be described. We assume that the human population is separated into six subpopulations, i.e. a general subpopulation $S(t)$, a semi-fanatical subpopulation $E(t)$, a fanatical subpopulation $F(t)$, an aware subpopulation $A(t)$, an imprisoned sub- population $P(t)$ and also a repented subpopulation $R(t)$. In this model, some fanatical people will be arrested at a rate of $\delta_{2}$ and separated into the subpopulation $P(t)$. Larger $\delta_{2}$ will also increase the rate of persuasion intensity from the fanatical subpopulation who have not been arrested, which is given by $\beta_{1}\left(1+\omega \delta_{2}\right)$, with $\omega$ as the worsening effect parameter of $\delta_{2}$. If $\delta_{2}=0$, then persuasion intensity is reduced to $\beta_{1}$. When fanatical people are arrested, they will receive counseling about the misdirection of their ideology from the government at a rate of $\eta$. With this counseling, some of them will separated into the $R(t)$ subpopulation which can persuade $S(t)$ to be aware of the ideology at a rate of $q \eta P(t)$, while others might come back to $F(t)$ at a rate of $(1-q) \eta P(t)$. To even up the comprehension strategy $\delta_{2}$, we also implement an intervention campaign about the danger of extreme ideology; let us call it $\delta_{1}$. In a real implementation, this intervention is related to how 
intensely the government provides education to the general and semi-fanatical populations to make them aware of the danger of this extreme ideology. With this intervention, some of the general and semi-fanatical subpopulation will be transferred to the aware subpopulation. To explain in more detail about the flow between subpopulations, we give the transition diagram in Figure 1.

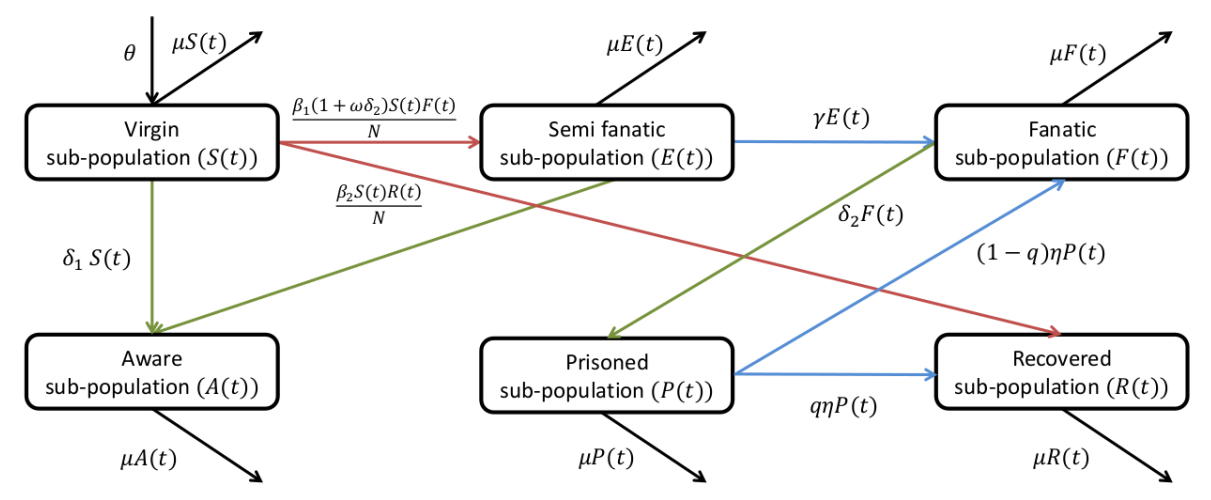

Figure 1: Transmission process of model 1

With the above assumptions, the mathematical model to explain the spread of the rumor with worsening effect of intervention is given by

$$
\left.\begin{array}{rl}
\frac{d S(t)}{d t} & =\theta-\frac{S(t)\left(\beta_{1}\left(1+\omega \delta_{2}\right) F(t)+\beta_{2} R(t)\right)}{N}-\left(\delta_{1}+\mu\right) S(t) \\
\frac{d E(t)}{d t} & =\frac{\beta_{1}\left(1+\omega \delta_{2}\right) S(t) F(t)}{N}-\left(\delta_{1}+\gamma+\mu\right) E(t) \\
\frac{d F(t)}{d t} & =\gamma E(t)+(1-q) \eta P(t)-\left(\delta_{2}+\mu\right) F(t) \\
\frac{d A(t)}{d t} & =\delta_{1}(S(t)+E(t))-\mu A(t) \\
\frac{d P(t)}{d t} & =\delta_{2} F(t)-q \eta P(t)-(1-q) \eta P(t)-\mu P(t) \\
\frac{d R(t)}{d t} & =\frac{\beta_{2} S(t) R(t)}{N}+q \eta P(t)-\mu R(t)
\end{array}\right\},
$$

with all parameter descriptions and their range of values given in Table 1.

\section{Mathematical Model Analysis}

In this section, mathematical model analysis of the model 1 to find the equilibrium points and their local stability will be performed. Since we assume 


\begin{tabular}{|c|l|c|}
\hline Var./Par. & Description & Value \\
\hline$S(t)$ & Virgin subpopulation & $S(t) \geq 0$ \\
$E(t)$ & Semi fanatic subpopulation & $E(t) \geq 0$ \\
$F(t)$ & Fanatic subpopulation & $F(t) \geq 0$ \\
$A(t)$ & Aware subpopulation & $A(t) \geq 0$ \\
$P(t)$ & Prisoned subpopulation & $P(t) \geq 0$ \\
$R(t)$ & Recovered subpopulation & $R(t) \geq 0$ \\
$\theta$ & New born recruitment rate & $N$ \\
$N$ & Total population & $\overline{65 \times 365}$ \\
$\beta_{1}$ & persuasiveness rate for $F(T)$ & $N \geq 0$ \\
$\beta_{2}$ & Persuasiveness rate for $R(T)$ & {$[0,1]$} \\
$\delta_{1}$ & Campaigns intervention rate & {$[0,1]$} \\
$\delta_{2}$ & Imprisonment intervention rate & {$[0,1]$} \\
$\omega$ & Worsening effect of $\delta_{2}$ & 0.1 \\
$\mu$ & Natural death rate & 0.1 \\
$\gamma$ & Transition rate from E(t) to $\mathrm{F}(\mathrm{t})$ & $\frac{1}{65 \times 365}$ \\
$\eta$ & Duration of prisoning time & 0.3 \\
$q$ & Portion of $P(t)$ repented from ideology & $\frac{1}{30 \times 365}$ \\
\hline
\end{tabular}

Table 1: Variables and parameters used in system 1 and their description. All variables and parameters are assumed to be non negative.

that the total of the human population is constant, from model 1 we see that $\frac{d N}{d t}=\theta-\mu N$ which will lead us to $N=\frac{\theta}{\mu}$.

Assuming the dynamic of each subpopulation in equation 1 equals zero, we find three different equilibrium points. the first equilibrium point represents when only the general subpopulation $(S(t))$ and aware subpopulation $(A(t))$ are established in the system, while other sub populations are zero. This equilibrium point is given by

$$
\Psi_{1}=\left(S_{1}, E_{1}, F_{1}, A_{1}, P_{1}, R_{1}\right)=\left(\frac{\theta}{\delta_{1}+\mu}, 0,0, \frac{\delta_{1} \theta}{\mu\left(\mu+\delta_{1}\right)}, 0,0\right) .
$$

This equilibrium point $\Psi_{1}$ will always be non-negative and always exist.

The second equilibrium point represents when only the general sub- population $S(t)$, the aware subpopulation $A(t)$ and the repented subpopulation $R(t)$ are established in the system, while the rest of the subpopulations are zero. This second equilibrium is given by:

$$
\Psi_{2}=\left(S_{2}, E_{2}, F_{2}, A_{2}, P_{2}, R_{2}\right)=\left(\frac{\theta}{\beta_{2}}, 0,0, \frac{\delta_{1} \theta}{\beta_{2} \mu}, 0, \frac{\theta\left(\beta_{2}-\delta_{1}-\mu\right)}{\mu \beta_{2}}\right) .
$$


As can be seen above, $\Psi_{2}$ is non-negative as long as

$$
L_{1}=\frac{\beta_{2}}{\delta_{1}+\mu}>1 .
$$

The last equilibrium point represents when all subpopulations are positive. This third equilibrium point unfortunately (let call it as $\Psi_{3}$ ) is not in simple form to be shown in explicit form, but for numerical purposes, the existence of this equilibrium point can still be shown.

The existence of the equilibrium $\Psi_{3}$ depend on the values of $F$ and $R$ given by

$$
\begin{aligned}
S & =\frac{\theta K}{R \beta_{2} \mu(\eta+\mu)} \\
E & =\frac{\beta_{1}\left(\omega \delta_{2}+1\right) K F}{R \beta_{2}(\eta+\mu)\left(\delta_{1}+\gamma+\mu\right)} \\
A & =\frac{\delta_{1} K\left(F \beta_{1} \mu \omega \delta_{2}+F \beta_{1} \mu+\gamma \theta+\mu \theta+\theta \delta_{1}\right)}{R \beta_{2} \mu^{2}(\eta+\mu)\left(\delta_{1}+\gamma+\mu\right)} \\
P & =\frac{\delta_{2} F}{\eta+\mu},
\end{aligned}
$$

with $K=\left(-F \eta q \delta_{2}+R \eta \mu+R \mu^{2}\right)$ while $F$ and $R$ are obtained from the solution of a coupled quadratic polynomial, given by

$$
\begin{aligned}
H_{1}(F, R)= & -F\left(F \gamma \beta_{1} \eta q \delta_{2}\left(\omega \delta_{2}+1\right)-\gamma R \beta_{1} \mu\left(\omega \delta_{2}+1\right)\right. \\
& \left.(\eta+\mu)+\beta_{2} R\left(\eta q \delta_{2}+\eta \mu+\mu^{2}+\mu \delta_{2}\right)\left(\delta_{1}+\gamma+\mu\right)\right), \\
H_{2}(F, R)= & \mu R(\eta+\mu)\left(R \beta_{2} \mu-\beta_{2} \theta+\mu \theta+\theta \delta_{1}\right) \\
& +F R \mu\left(\beta_{1} \mu\left(\omega \delta_{2}+1\right)(\eta+\mu)-\beta_{2} \eta q \delta_{2}\right) \\
& -F^{2} \beta_{1} \eta \mu q \delta_{2}\left(\omega \delta_{2}+1\right)-F \eta q \theta \delta_{2}\left(\delta_{1}+\mu\right) .
\end{aligned}
$$

By substituting the parameter values, the existence of $F$ and $R$ can be shown numerically in Figure 2. If there is intersection between $H_{1}$ and $H_{2}$ in the first quadrant, we can say that a third equilibrium point $\left(\Psi_{3}\right)$ exists.

To investigate the local stability of equilibrium points mentioned above, first we find the Jacobian matrix $(\mathcal{J})$ of system 1 which is given in the form 


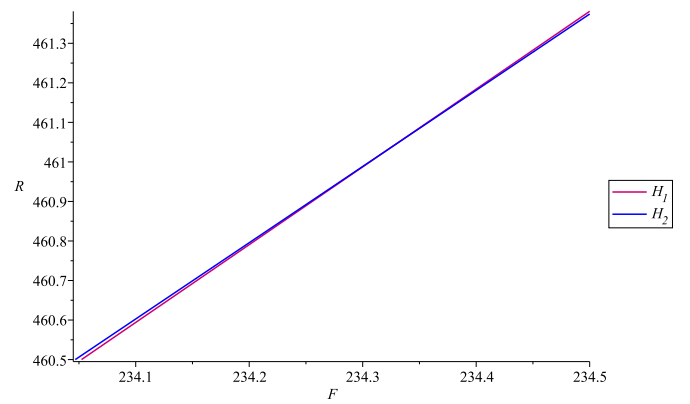

Figure 2: Existence of endemic equilibrium for $F$ and $R$.

of:
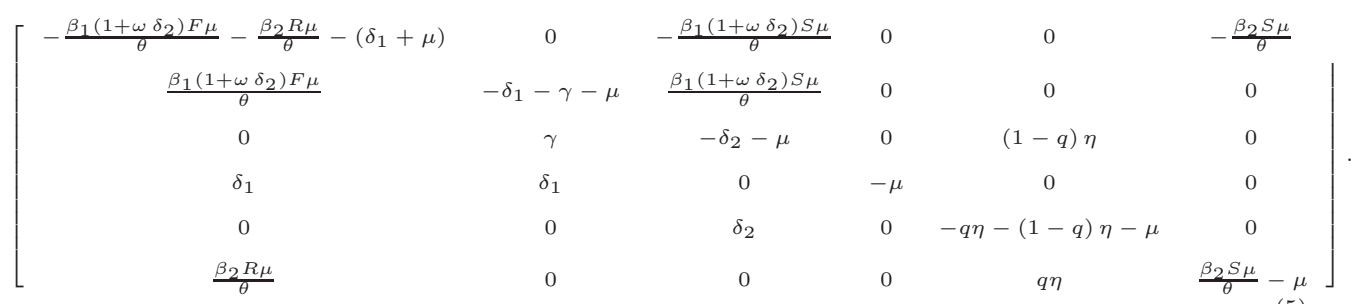

(5)

Evaluate equilibrium point $\Psi_{1}$ in Jacobian matrix $\mathcal{J}$ give us

$$
\mathcal{J}_{1}=\left[\begin{array}{cccccc}
-\delta_{1}-\mu & 0 & -\frac{\beta_{1}\left(1+\omega \delta_{2}\right) \mu}{\delta_{1}+\mu} & 0 & 0 & -\frac{\beta_{2} \mu}{\delta_{1}+\mu} \\
0 & -\delta_{1}-\gamma-\mu & \frac{\beta_{1}\left(1+\omega \delta_{2}\right) \mu}{\delta_{1}+\mu} & 0 & 0 & 0 \\
0 & \gamma & -\delta_{2}-\mu & 0 & (1-q) \eta & 0 \\
\delta_{1} & \delta_{1} & 0 & -\mu & 0 & 0 \\
0 & 0 & \delta_{2} & 0 & -q \eta-(1-q) \eta-\mu & 0 \\
0 & 0 & 0 & 0 & q \eta & \frac{\beta_{2} \mu}{\delta_{1}+\mu}-\mu
\end{array}\right] .
$$

Equilibrium point $\Psi_{1}$ is locally asymptotically stable if all eigenvalues of $\mathcal{J}_{1}$ are negative. we have three explicit eigenvalues, i.e $\lambda_{1}=-\mu, \lambda_{2}=-\left(\delta_{1}+\mu\right)$ and $\lambda_{3}=-\frac{\mu\left(1-L_{1}\right)}{\delta_{1}+\mu}$. The other eigenvalues is taken from roots of

$$
D_{3} \lambda^{3}+D_{2} \lambda^{2}+D_{1} \lambda+D_{0}
$$

where

$D_{3}=\delta_{1}+\mu$ 


$$
\begin{aligned}
D_{2}= & \left(\delta_{1}+\mu\right)\left(\delta_{1}+\eta+3 \mu+\gamma+\delta_{2}\right) \\
D_{1}= & \left(2 \delta_{1} \mu+\delta_{1} \delta_{2}+\delta_{1} \eta+2 \mu \delta_{2}+2 \gamma \mu+3 \mu^{2}+\gamma \delta_{2}+\delta_{2} q \eta+\gamma \eta+2 \eta \mu\right) \\
& \left(\delta_{1}+\mu\right)-\gamma \beta_{1} \mu\left(1+\omega \delta_{2}\right) \\
D_{0}= & \left(\eta \mu+\mu^{2}+\mu \delta_{2}+\delta_{2} q \eta\right)\left(\delta_{1}+\mu\right)\left(\delta_{1}+\gamma+\mu\right) \\
& -\gamma \beta_{1} \mu(\mu+\eta)\left(1+\omega \delta_{2}\right)
\end{aligned}
$$

According to the Routh-Hurwitz stability criteria, all roots of 6 are negative if

$$
\begin{aligned}
\mathcal{K}_{0} & =\frac{\gamma \beta_{1} \mu(\mu+\eta)\left(1+\omega \delta_{2}\right)}{\left(\eta \mu+\mu^{2}+\mu \delta_{2}+\delta_{2} q \eta\right)\left(\delta_{1}+\mu\right)\left(\delta_{1}+\gamma+\mu\right)}<1 \\
\mathcal{K}_{1} & =\frac{\gamma \beta_{1} \mu\left(\omega \delta_{2}+1\right)}{\left(\delta_{1}+\mu\right)\left(\eta q \delta_{2}+\eta \gamma+2 \eta \mu+\eta \delta_{1}+2 \gamma \mu+\gamma \delta_{2}+3 \mu^{2}+2 \mu \delta_{1}+2 \mu \delta_{2}+\delta_{1} \delta_{2}\right)} \\
& <1 \\
\mathcal{K}_{2} & =\frac{D_{0} D_{3}}{D_{2} D_{1}}<1 .
\end{aligned}
$$

Using the same approach as with the first equilibrium, we evaluate the local stability criteria of $\Psi_{2}$ with the following Jacobian matrix

$$
\begin{aligned}
& \mathcal{J}_{2}= \\
& {\left[\begin{array}{cccccc}
-\beta_{2} & 0 & -\frac{\beta_{1}\left(1+\omega \delta_{2}\right) \mu}{\beta_{2}} & 0 & 0 & -\mu \\
0 & -\delta_{1}-\gamma-\mu & \frac{\beta_{1}\left(1+\omega \delta_{2}\right) \mu}{\beta_{2}} & 0 & 0 & 0 \\
0 & \gamma & -\delta_{2}-\mu & 0 & \eta(1-q) & 0 \\
\delta_{1} & \delta_{1} & 0 & -\mu & 0 & 0 \\
0 & 0 & \delta_{2} & 0 & -q \eta-\eta(1-q)-\mu & 0 \\
\beta_{2}-\delta_{1}-\mu & 0 & 0 & 0 & q \eta & 0
\end{array}\right] .}
\end{aligned}
$$

Again with the Routh-Hurwitz criteria, we will try to find the local stability criteria of $\Psi_{2}$. From the characteristic polynomial of $\mathcal{J}_{2}$, we find one explicit eigenvalue i.e. $\lambda_{1}=-\mu$, and two other eigenvalues $\lambda_{2}, \lambda_{3}$ will negative if and only if $L_{1}>1$. The other eigenvalues are taken from the characteristic polynomial of

$$
G_{3} \lambda^{3}+G_{2} \lambda^{2}+G_{1} \lambda+G_{0}=0
$$

where

$$
G_{3}=\beta_{1}
$$




$$
\begin{aligned}
G_{2}= & \beta_{2}\left(3 \mu+\gamma+\eta+\delta_{1}+\delta_{2}\right) \\
G_{1}= & 3 \beta_{2} \mu^{2}+2 \beta_{2}\left(\gamma+\delta_{1}+\delta_{2}+\eta\right) \mu-\gamma \beta_{1} \mu\left(1+\omega \delta_{2}\right) \\
& +\beta_{2}\left(\eta \gamma+\delta_{1} \eta+\delta_{1} \delta_{2}+q \eta \delta_{2}+\delta_{2} \gamma\right) \\
G_{0}= & \beta_{2}\left(\mu^{2}+q \eta \delta_{2}+\mu \delta_{2}+\mu \eta\right)\left(\mu+\delta_{1}+\gamma\right) \\
& -\gamma \beta_{1} \mu\left(1+\omega \delta_{2}\right)(\mu+\eta) .
\end{aligned}
$$

According to the Routh-Hurwitz stability criteria, all roots of $G$ will be negative if and only if

$$
\begin{aligned}
\mathcal{M}_{0} & =\frac{\gamma \beta_{1} \mu\left(1+\omega \delta_{2}\right)}{3 \beta_{2} \mu^{2}+2 \beta_{2}\left(\gamma+\delta_{1}+\delta_{2}+\eta\right) \mu+\beta_{2}\left(\eta \gamma+\delta_{1} \eta+\delta_{1} \delta_{2}+q \eta \delta_{2}+\delta_{2} \gamma\right)} \\
& <1 \\
\mathcal{M}_{1} & =\frac{\gamma \beta_{1} \mu\left(\omega \delta_{2}+1\right)(\eta+\mu)}{\beta_{2}\left(q \eta \delta_{2}+\eta \mu+\mu^{2}+\mu \delta_{2}\right)\left(\delta_{1}+\gamma+\mu\right)} \\
& <1 \\
\mathcal{M}_{2} & =\frac{G_{0} G_{3}}{G_{1} G_{2}}<1 .
\end{aligned}
$$

\section{Numerical Simulation}

For simulation in this chapter, we show that the existence of the three equilibrium points depends on several conditions (Figure 3 ). The parameter values for this simulation are given in Table ??.

\begin{tabular}{|c|c|}
\hline Parameter & Value \\
\hline$N$ & 1000 \\
$\theta$ & $\frac{1000}{65 \times 365}$ \\
$\mu$ & $\frac{1}{65 \times 365}$ \\
$\eta$ & $\frac{1}{15 \times 365}$ \\
$\gamma$ & $\frac{1}{30}$ \\
$q$ & 0.5 \\
$w$ & 1 \\
$\beta_{1}$ & 0.6 \\
$\beta_{2}$ & 0.01 \\
\hline
\end{tabular}

Table 2: Parameter value 

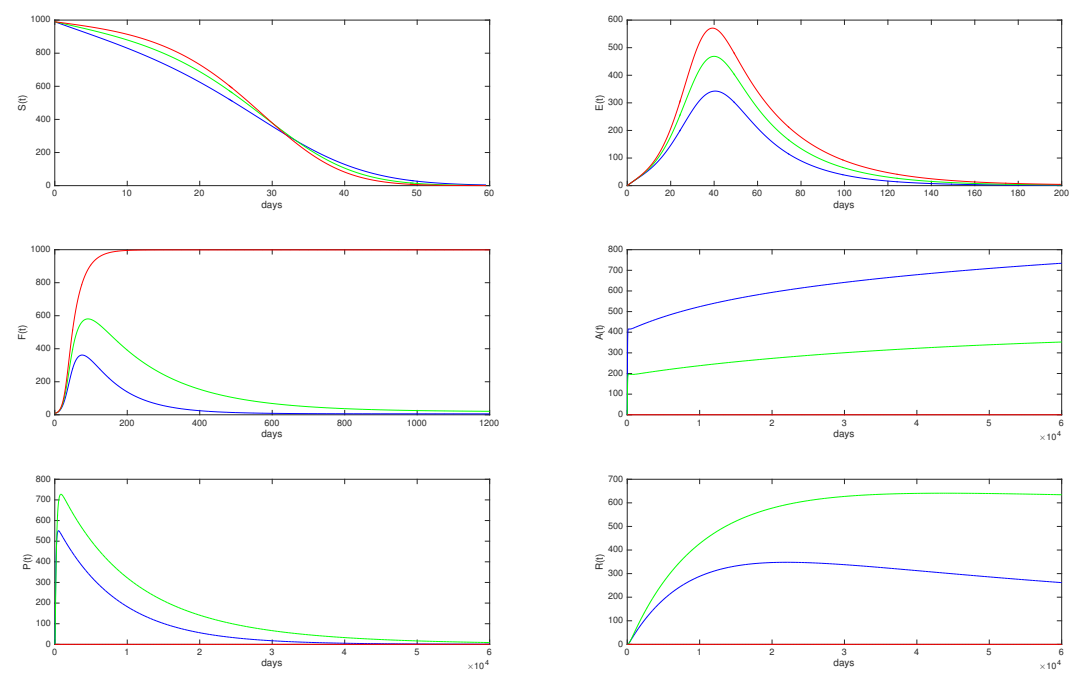

Figure 3: Comparing three different conditions depend on $\delta_{1}$ and $\delta_{2}$. We use blue curve for $\delta_{1}=0.01, \delta_{2}=0.01$, green curve for $\delta_{1}=0.004, \delta_{2}=$ 0.005 , and red curve for $\delta_{1}=0, \delta_{2}=0$

The first condition is given by the red curve when there is no intervention from the government. It means there is no campaign about the dangers of the rumor $\left(\delta_{1}=0\right)$ and no imprisonment of fanatical people $\left(\delta_{2}=0\right)$. From the first row in Figure 3, we can see that the population will become fanatical people. In contrast, when there is intervention from the government which is shown by the green curve $\left(\delta_{1}=0.004 ; \delta_{2}=0.005\right)$ and the blue curve $\left(\delta_{1}=0.01\right.$ $\left.; \delta_{2}=0.01\right)$, it can be seen that the impact of the government campaign and imprisonment will decrease the size of the fanatical population also increase the size of the aware and repented population.

\begin{tabular}{|c|c|c|c|c|c|c|c|c|}
\hline \multicolumn{2}{|c|}{ Parameter } & \multicolumn{3}{|c|}{ Stability criteria $\psi_{1}$} & \multicolumn{3}{|c|}{ Stability criteria $\psi_{2}$} & \multirow{2}{*}{$\begin{array}{r}\text { Stable } \\
\text { Point }\end{array}$} \\
\hline$\delta_{1}$ & $\delta_{2}$ & $\mathcal{K}_{0}$ & $\mathcal{K}_{1}$ & $\mathcal{K}_{2}$ & $\mathcal{M}_{0}$ & $\mathcal{M}_{1}$ & $\overline{\mathcal{M}_{2}}$ & \\
\hline 0 & 0 & 14217 & 2242 & $6 \times 10^{-3}$ & 9.45 & 59.92 & $6 \times 10^{-2}$ & $\Psi_{3}$ \\
\hline $4 \times 10^{-3}$ & $5 \times 10^{-3}$ & 1.86 & 1.06 & $4 \times 10^{-2}$ & 0.42 & 0.75 & $10^{-3}$ & $\Psi_{2}$ \\
\hline $10^{-2}$ & $10^{-2}$ & 0.33 & 0.19 & $2 \times 10^{-3}$ & 0.19 & 0.32 & $2 \times 10^{-3}$ & $\Psi_{1}$ \\
\hline
\end{tabular}

Table 3: Existence and stability criteria for equilibrium points

A simulation of how the arrest of the fanatical population will worsen the 


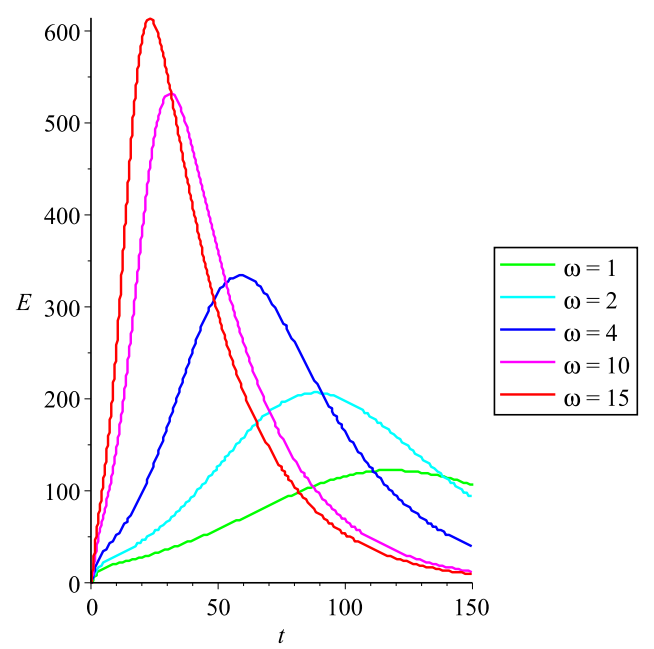

(a)

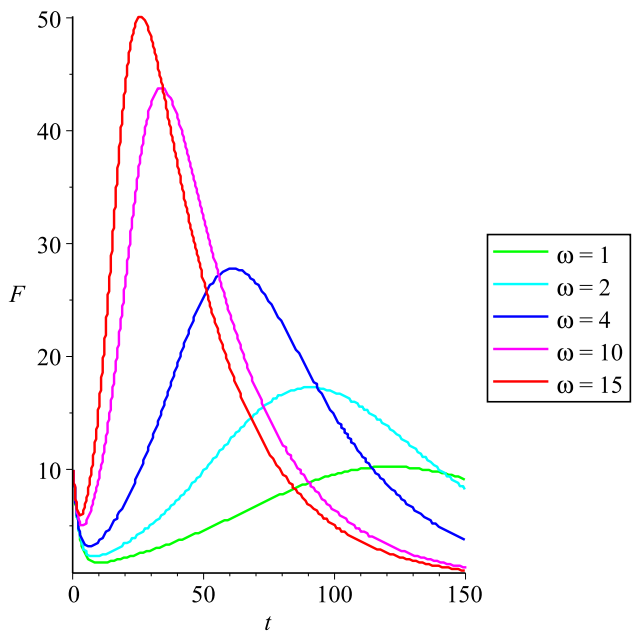

(b)

Figure 4: Sensitivity $\omega$ respect to number of (a) semi-fanatic population (b) fanatic population

spread of a rumor is shown in Figure 4. We assume that $40 \%$ of fanatical population are imprisoned. Imprisonment could increase sympathy among fellow fanatics, so the population that has not been caught will be more active in spreading their ideology. Worsening parameter $(\omega)$ affects the dynamics of the fanatical population. Even though in the end the fanatical population will disappear, a large value of $\omega$ will cause a big number of fanatical people to occur faster and will increase the size of the fanatical population more compared with a small value of $\omega$.

The next simulation is performed to show the sensitivity of $\delta_{1}$ which represents the effect of government campaign about the dangers of the rumor. In this case, we assume that there are only a few fanatical people who can be arrested and imprisoned $\left(\delta_{2}=0.0005\right)$. From Figure 5, we can see that a larger value of $\delta_{2}$ will increase the size of the aware population (see Figure 5 (top) ) and decrease the size of the fanatical population (see Figure 5 (bottom)). This means the government must campaign more intensely so that more people will be aware that the ideology is dangerous and they will stay away from the fanatical population. 

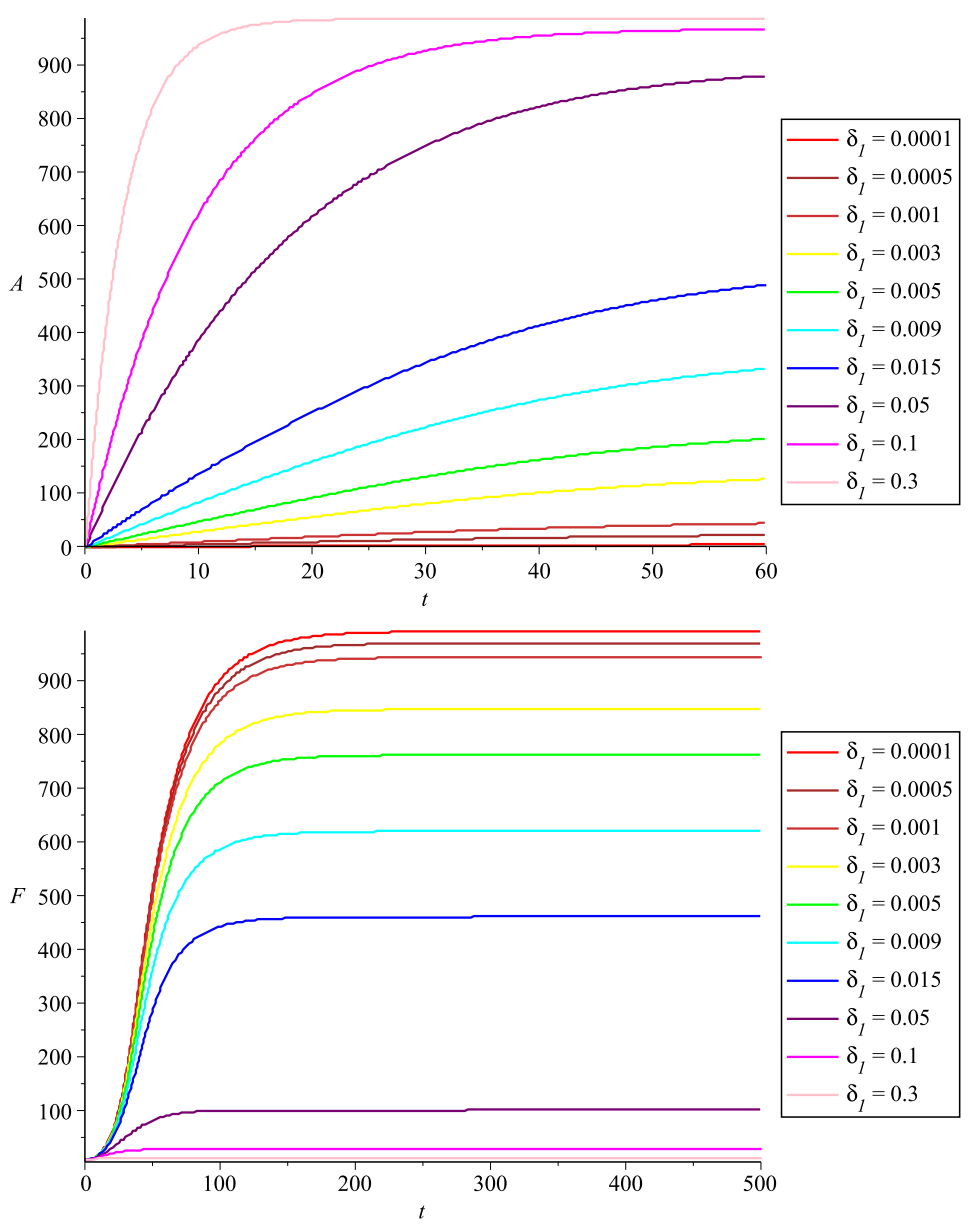

Figure 5: Sensitivity $\delta_{1}$ respect to number of (a) fanatic population (b) aware population

\section{Conclusions}

A mathematical model to demonstrate the spread of a rumor is constructed in this article. Interventions by government to suppress the spread of the rumor can be implemented in two ways, i.e. through a campaign about the dangers of the rumor and also by restraining the fanatical population. Unfortunately, restraint techniques will have a worsening effect by increasing the size of the fanatical population to persuade general population.

Mathematical analysis to find all equilibrium points and local stability has 
been shown. We have three equilibrium points, i.e. when only a general and an aware sub population exist; only a general, aware and a repented sub population exist; and a coexistence equilibrium. From the numerical simulation, it is shown that an intervention using campaign should be consider wisely since it will accelerate fanatical population persuasion to general population to follow their ideology. As consideration of the high worsening effect, even though the size of the fanatical population will continue to decline toward 0 , the fanatical population will reach a critical condition before reaching 0 .

\section{Acknowledgments}

We would like to thank all the referee for all suggestions and comments for this article. This research is funded by the Ministry of research, technology and Higher Education 2016, Indonesia.

\section{References}

[1] D.Aldila, N.Nuraini, E.Soewono, Mathematical model for the spread of extreme ideology, AIP Conf. Proc., 1651, No. 33 (2015), 33-39.

[2] R. Jie, J. Qiao, G. Xu, Y. Meng, A study on the interaction between two rumors in homogeneous complex networks under symmetric conditions, Physica A: Statistical Mechanics and its Applications, 454, (2016), 129-142 .

[3] M.Z.Dauhoo, D.Juggurnath, N-R. B. Adam, The stochastic evolution of rumors within a population, Mathematical Social Sciences, 82, (2016), 85-96.

[4] L.Zhu, H.Zhao, H.Wang, Complex dynamic behavior of a rumor propagation model with spatial-temporal diffusion terms, Information Sciences, 349-350, (2016), 119-136

[5] L. Huo, J. Jiang, S. Gong, B. He, Dynamical behavior of a rumor transmission model with Holling-type II functional response in emergency event, Physica A: Statistical Mechanics and its Applications, 450, (2016), 228-240.

[6] L. Zhao, H. Cui, X. Qiu, X. Wang, J. Wang, SIR rumor spreading model in the new media age, Physica A: Statistical Mechanics and its Applications, 392, No. 4 (2013), 9951003.

[7] G. Chen, H. Shen, T. Ye, G. Chen, N. Kerr, A kinetic model for the spread of rumor in emergencies, Discrete Dynamics in Nature and Society, 2013, No. 605854 (2013), 1-8 .

[8] D.J.Dalley and .G.Kendall, Stochastic rumours, IMA Journal of Applied Mathematics , 1, (1965), 4255.

[9] K. Kawachi, M. Seki, H. Yoshida, Y. Otake, K. Warashina, H. Ueda, A rumor transmission model with various contact interactions, Journal of Theoritical Biology, 253, No. 1 (2008), 55-60. 